\title{
Changing clinical characteristics of non- cystic fibrosis bronchiectasis in children
}

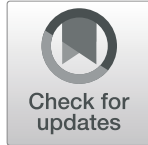

\author{
Ela Erdem Eralp ${ }^{1 *}$ (D), Yasemin Gokdemir ${ }^{1}$, Emine Atag ${ }^{1}$, Nilay Bas Ikizoglu', Pinar Ergenekon ${ }^{1}$, Cansu Yilmaz Yegit ${ }^{1}$, \\ Arif Kut ${ }^{2}$, Refika Ersu', Fazilet Karakoc ${ }^{1}$ and Bulent Karadag ${ }^{1}$
}

\begin{abstract}
Background: The prevalence of non-cystic fibrosis (CF) bronchiectasis is increasing in both developed and developing countries in recent years. Although the main features remain similar, etiologies seem to change. Our aim was to evaluate the clinical and laboratory characteristics of our recent non-CF bronchiectasis patients and to compare these with our historical cohort in 2001.
\end{abstract}

Methods: One hundred four children with non-CF bronchiectasis followed between 2002 and 2019 were enrolled. Age of diagnosis, underlying etiology and microorganisms in sputum culture were recorded. Clinical outcomes were evaluated in terms of lung function tests and annual pulmonary exacerbation rates at presentation and within the last 12 months.

Results: Mean FEV1 and FVC \%predicted at presentation improved compared to historical cohort (76.6 \pm 17.1 vs. $63.3 \pm 22.1$ and $76.6 \pm 15.1$ vs. $67.3 \pm 23.1$, respectively; $p<0.001$ ). There was a significant decrease in pulmonary exacerbation rate from $6.05 \pm 2.88$ at presentation to $3.23 \pm 2.08$ during follow-up $(p<0.0001)$. In $80.8 \%$ of patients, an underlying etiology was identified. There was an increase in primary ciliary dyskinesia (PCD) (32.7\% vs. $6.3 \% ; p=0.001)$, decrease in idiopathic cases (19.2\% vs. $37.8 \% ; p=0.03)$ with no change in postinfectious and immunodeficiencies as underlying etiology. Sputum cultures were positive in $77.9 \%$ of patients which was $46.9 \%$ in the historical cohort ( $p=0.001$ ). Conclusion: Baseline pulmonary function tests were better and distribution of underlying etiology had changed with a remarkable increase in diagnosis of PCD in the recent cohort.

Keywords: Bronchiectasis, Child, Primary ciliary dyskinesia, Spirometry

\section{Background}

Childhood bronchiectasis is a chronic pulmonary disorder defined as a clinical syndrome (persistent or recurrent [>3] episodes of chronic wet or productive cough, sometimes with coarse crackles and digital clubbing), confirmed by the presence of bronchial dilation in high resolution chest tomography (HRCT) [1]. Although bronchiectasis has been called as an orphan disease in the past, it is now considered as one of the most

\footnotetext{
* Correspondence: elaerdem@yahoo.com

'Division of Pediatric Pulmonology, Marmara University, School of Medicine, Istanbul, Turkey

Full list of author information is available at the end of the article
}

common causes of chronic respiratory disease in both developed and developing countries [2].

The exact incidence of non-cystic fibrosis (CF) bronchiectasis in children is not known, but studies from England and New Zealand people of European heritage reported the incidence as $0.2 / 100,000$ and 1.5/100,000 per year respectively; whereas incidence was highest in Pacific children as 17.8/100.000 [3, 4]. Main etiologies for non-CF bronchiectasis are infections, immune deficiencies, primary ciliary dyskinesia (PCD), and aspirations, but in $34 \%$ of patients no underlying cause was identified [5]. Both in developed and developing countries, postinfection was the leading etiology of non-CF 
bronchiectasis in the past years [3]. Highest incidences were reported in socially disadvantaged populations of developed countries as $90 \%$ by Chang et al. from Australia and $93 \%$ by Singleton et al. from Alaska [6, 7]. Brower et al. in a review including 12 studies found recurrent lower respiratory tract infections as the most common underlying cause (61\%), followed by measles (14\%), tuberculosis (11\%) and pertussis (5\%) [5]. With improvement in vaccination programs, prevention of diseases like measles and pertussis, effective tuberculosis control programs, improved access to health care and effective treatment of lower respiratory tract infections incidence of postinfectious bronchiectasis decreased to $7-12 \%$ in developed countries [8-10]. Immune deficiencies (10$34 \%)$ and PCD (2-24\%) as the underlying reason were generally reported more frequently in developed countries with no change in the frequency over the years [8-13]. Idiopathic cases did not seem to change in frequency in both developed and developing countries in the last years. Kapur et al. in a recent study found no underlying etiology in $55 \%$ of patients in a developed country [9]. Also in a study conducted with 80 children from India by Kumar et al., 36\% of cases were classified as idiopathic [14].

Although there is a decrease in post infectious bronchiectasis in developed countries, overall prevalence of non-CF bronchiectasis appears to be increasing particularly in adults $[15,16]$. This increase in prevalence may be related to improved diagnostic rates secondary to improved awareness and improved access to HRCT scans.

We previously reported clinical features of non-CF bronchiectasis followed in our division between 1987 and 2001 [17]. To our knowledge, changes in general characteristics of patients with non-CF bronchiectasis in time in the same center have not been reported previously. We hypothesized that the underlying etiology and clinical characteristics of non-CF bronchiectasis changed over time. Our aim was to evaluate the changing characteristics of non-CF bronchiectasis in a decade comparing the recent cohort of patients with historical controls.

\section{Methods}

This was an observational study including two cohorts from the same center. Recent cohort consisted of nonCF bronchiectasis patients ( $<18$ years of age) who have been followed in the Marmara University Pediatric Pulmonology clinic between 2002 and 2019. Historical cohort consisted of patients enrolled in our previous study which was done between 1987 and 2001. There were no overlapping patients between two cohorts. Study was approved by the local ethics committee and written informed consent was taken from the families.

Patients with a clinical history of recurrent or persistent productive cough, sputum, wheeze, breathlessness unresponsive to treatment and recurrent lower respiratory tract infections were evaluated for bronchiectasis (Fig. 1) [18]. Diagnosis of bronchiectasis confirmed by the HRCT obtained during an asymptomatic period and at least 3 months after the last infectious exacerbation. Bronchiectasis was diagnosed if there was evidence of bronchial dilation (internal bronchial diameter greater than the accompanying pulmonary artery) and a lack of bronchial tapering on sequential slices [19]. The bronchi were evaluated on a lobar basis (regarding the lingula as a separate lobe). Bronchiectasis was defined as localized if only one lobe, and multilobar if more than one lobe was was affected.

\section{Study design}

Patients were followed up at 3 months intervals. At each visit, patients or parents were asked about the presence of symptoms (cough, sputum, wheezing, dyspnea, hemotysis) and frequency of antibiotic use since last visit. Demographic, clinical and laboratory data including gender, age at the of onset of symptoms, duration of symptoms before diagnosis, age at diagnosis, presence of consanguinity, presenting symptoms; history of previous lower respiratory infections including pertussis, measles, varicella, tuberculosis before the diagnosis; number of lower respiratory tract infections within the previous 12 months at presentation and within the last 12 months of the follow-up requiring oral or intavenous antibiotics or hospitalization, history of surgery for bronchiectasis, localization of bronchiectasis on chest $\mathrm{CT}$ scan and pulmonary fuction test results were retrieved from medical records. Pulmonary exacerbation was defined by the any of the following; change in cough quality from dry to wet and/or sputum production for $\geq 3$ days, breathlessness, chest pain, crepitations, wheeze with or without an increase in values of infectious markers [20, 21]. The presence of clubbing, chest deformities and nasal polyps were noted on physical examination. For microbiological evaluation, sputum samples were obtained when possible and cultured for bacteria.

For the etiological work-up of bronchiectasis, immunological evaluation including IgG, M, A and E, IgG subclass levels, specific antibody responses to tetanus toxoid, capsular polysaccharides of Streptococcus pneumonia and surface antigen of Hepatitis B virus, $T$ lymphocyte subsets and neutrophil function tests were measured in all patients. In order to exclude CF, sweat test and genetic analysis (if required) were performed and patients with positive sweat tests (chloride levels $>60 \mathrm{mEq} / \mathrm{l}$ ) or two CF mutations were excluded from the study.

Postinfectious etiology was evaluated by asking history of lower respiratory infections including measles, pertussis, varicella and tuberculosis. We performed tuberculin skin test (TST) to on all patients and patients with positive TST were re-evaluated further for tuberculosis.

PCD was diagnosed by the electronic microscopic evaluation of nasal cilia biopsy and/or positive 


\section{Symptoms}

- Clinical history of recurrent or persistent productive cough, sputum, wheeze, breathlessness unresponsive to treatment $>4$ weeks,

- Recurrent lower respiratory tract infections,

- Clubbing

- Hemoptysis

- Persistent pneumonia or chest radiograph changes

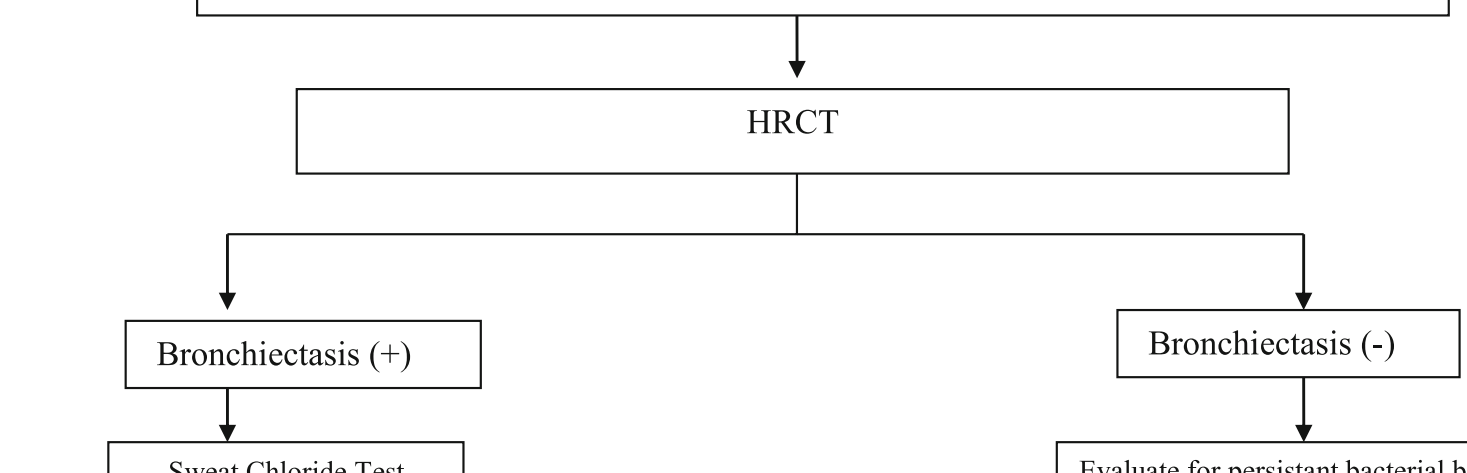

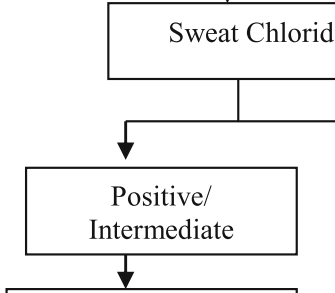

Evaluate for $\mathrm{CF}$

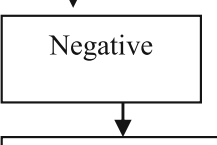

-Spirometry in children age $>6$ years -Sputum culture, TST

-Evaluate for immune deficiencies

-Evaluate for gastroesophageal reflux and aspiration

-Evaluate for PCD

-Flexible bronchoscopy in localized bronchiectasis

\begin{tabular}{|l|}
\hline \multicolumn{1}{|c|}{ Management of patient with non-CF } \\
\hline 1-Chest physiotherapy with inhaled \\
bronchodilator \\
2-Inhaled corticosteroid in patients with \\
bronchial hyperreactivity \\
3-Prophylactic azithromycin 3 days/week in \\
patients with frequent exacerbations. \\
4-Treatment for asssociated co-morbidities \\
(GER, allergic rhinitis, etc) \\
5- Treatment for the underlying disease \\
(immune deficiency, GER, foreign body \\
aspiration) \\
5- Assssment and managemnt of nutrition \\
5- Treatment of infections: Oral/IV antibiotics \\
for pulmonary exacerbations \\
6- Seasonal influenza and pneumoccal vaccine \\
7- Surgery (in certain circumstances)
\end{tabular}

Evaluate for persistant bacterial bronchitis If the symptoms persist:

-Spirometry

-Sputum culture, TDT

-Evaluate for immune deficiencies

-Evaluate for gastroesophageal reflux and aspiration

-Evaluate for nutritional status

-Evaluate for PCD

-Flexible bronchoscopy

Fig. 1 Algorithm for diagnosis and treatment of non-CF bronchiectasis 
immunofluorescence staining and/or decreased nasal nitric oxide level measurement and/or the presence of dextrocardia with the typical findings of PCD and PICADAR score $>5$. Nasal nitric oxide cutoff value for PCD was defined at $77 \mathrm{nl} /$ minute [22]. In patients with a decreased nasal nitric oxide levels but a negative electronic microscopic result or in whom evaulation could not be done, immunofluorescence staining was performed in Muenster University Hospital. Antibodies against DNAH5, GAS8, DNAH11 and RSPH9; in some selected cases DNALI1 and CCDC39 were asessed.

Asthma was diagnosed if patients had the history of wheeze, shortness of breath, chest tightness and/or cough and by variable expiratory airflow limitation according to Global Initiative for Asthma (GINA) [23]. In order to differentiate asthma from bronchiectasis patients with bronchial hyperreactivity, patients followed at least for 2 years before the diagnosis of bronchiectasis in a pediatric allergy or pulmonology clinic without any abnormality in chest $\mathrm{x}$ ray or HRCT before, were labelled as asthmatic. Patients without an underlying etiology for bronchiectasis were classified as idiopathic bronchiectasis.

Spirometry (MIR Srl Spirobank, Rome, Italy) was performed according to the criteria of the American Thoracic Society by all children who were able to cooperate. Measurements included forced vital capacity (FVC) and forced expiratory volume in $1 \mathrm{~s}$ (FEV1); values were expressed as percentage of the predicted normal values [24]. Measurements were repeated $15 \mathrm{~min}$ after inhalation of 200 microgram salbutamol metered dose inhaler via a spacer (Volumatic, Allen \& Hanburys, Uxbridge, UK). A positive bronchodilator response was defined as an FEV1 improvement of more than $12 \%$ of baseline [25].

\section{Statistical analysis}

Statistical analysis was carried out with with SPSS for Windows version 20.0. Continuous variables were described through means, standard deviations and medians, whereas categorical variables were presented as frequency and percentages. Categorical variables were compared with Chi-square or with Fisher's exact test when $20 \%$ of the expected frequencies were less than five. Continuous variables among two groups were compared with Mann-Whitney U test, since the data did not follow a normal distribution. Continous variables for paired groups were compared through Wilcoxon test. Results were evaluated in 95\% confidence interval and significance level was set at $p$ value of 0.05 .

\section{Results}

\section{Demographic characteristisc}

Recent cohort included 104 patients diagnosed as bronchiectasis after 2002 (45\% male) with a median age of 8 years (range, $0.1-16.5)$ at presentation.
In the recent cohort, median age of diagnosis was 7 years. The most common presenting symptom was cough (95.2\%) followed by sputum (77.9\%), wheezing (42.3\%) and dyspnea (51\%). Table 1 shows the general characteristics of two cohorts. Rate of clubbing was decreased, baseline $\mathrm{FEV}_{1}$ and $\mathrm{FVC}$ values were higher in the recent cohort when compared to the historical one (Table 1).

In terms of pulmonary function, there was an increase in the mean $\mathrm{FEV}_{1}$ and FVC $(p<0.001)$ in the recent cohort. Similar to the historical cohort, annual lower respiratory tract infection rate decreased from $6.1 \pm 2.9$ at presentation to $3.2 \pm 2.1$ during follow-up $(p<0.0001)$. There was no change between the baseline and last $\mathrm{FEV}_{1} \%$ in follow-up in the recent cohort (76.6 $\pm 17.1 \mathrm{vs}$. $76.96 \pm 18.1 ; p=0.91)$ which increased in the historical one $(63.3 \pm 22.1$ vs. $75.2 \pm 25.2 ; p<0.001)$.

\section{Etiology}

Underlying etiology was identified in $80.8 \%$ (n:84) of the patients in the resent cohort (Table 2). Most common cause of non-CF bronchiectasis was PCD (32.7\%), followed by post-infectious (26\%) and immunodeficiencies (17.3\%). All of the PCD patients (n:34) had typical findings of PCD and PICADAR score $>5$. In 26 of patients nasal nasal nitric oxide measurement, in 4 transmission electron microscopy, in 19 immunofluorescence staining (7 with outer dynein arm defect, 4 with inner dynein arm defect, 4 with nexin-dynein regulatory complex defect and 2 with radial spoke defects) and in 4 genetic analysis (2 patients had CCDC40 homozygous, 1 CCNO homozygous and RSPH4A homozygous mutations) were performed [26]. There was a significant increase in the frequency of $\operatorname{PCD}(6.3 \%$ vs. $32.7 \%, p=$ $0.001)$, and decrease in idiopathic cases $(37.8 \%$ vs. $19.2 \%$, $p=0.03)$ compared with the historical cohort. In the recent cohort, there was no patient with a history of foreign body aspiration. One patient with esophageal atresia and tracheoesophageal fistula and two patients operated for complex cardiac disease were classified in other group.

\section{Localization of bronchiectasis}

Localization of bronchiectasis was similar to the historical cohort, and left lower lobe was the most affected lobe in both groups. In $38.5 \%$ of the patients there was one lobe involvement, mostly the left lower lobe (21.2\%) similar to the historical cohort $(p=0.75)$. Although statistically insignificant, multilobar involvement tended to decrease (31.9\% vs. $21.4 \%, p=0.26)$ and bilobar involvement trended to increase $(28.1 \%$ vs $41.3 \%(p=0.09)$ in the recent cohort compared with the historical one. 
Table 1 Demographic data for non-CF patients

\begin{tabular}{|c|c|c|c|}
\hline & Historical Cohort 1987-2001 (n:111) & Recent Cohort 2002-2019 (n:104) & $p$-value \\
\hline Male gender $n(\%)$ & $56(50.5)$ & $47(45)$ & 0.52 \\
\hline Age of onset of symptoms (yr), median (min-max) & $1.5(0-11.9)$ & $1(0-16)$ & 0.11 \\
\hline \multicolumn{4}{|l|}{ Duration of symptoms before diagnosis (yr), } \\
\hline median (min-max) & $4(0.1-14.9)$ & $4(0-15.5)$ & 0.89 \\
\hline Age at presentation (yr), median (min-max) & $7(1-17.5)$ & $8(0.1-16.5)$ & 0.36 \\
\hline Hemoptysis n(\%) & $11(10)$ & $7(6.7)$ & 0.40 \\
\hline Clubbing $n(\%)$ & $45(40.9)$ & $22(21.2)$ & 0.02 \\
\hline Chest deformity,n(\%) & $16(14.5)$ & $12(11.5)$ & 0.53 \\
\hline Annual lower respiratory tract infection rate (mean \pm SD) & $6.6 \pm 4.0$ & $6.1 \pm 2.9$ & 0.91 \\
\hline \multicolumn{4}{|l|}{ Spirometry n:93 (mean \pm SD) } \\
\hline FEV1 (\% predicted) & $63.3 \pm 22.1$ & $76.6 \pm 17.1$ & $<0.001$ \\
\hline FVC (\% predicted) & $67.3 \pm 23.1$ & $76.7 \pm 15.1$ & $<0.001$ \\
\hline
\end{tabular}

\section{Sputum culture}

Rate of positive sputum culture increased in the recent cohort (46.9\% vs. $77.9 \%, p=0.001)$. Most frequently isolated organisms were H.influenza (71.8\%), S.pneumonia (47.1\%), M.catarrhalis (14.4\%), P.aeruginosa (11.5\%) and S.aureus $(11.5 \%)$, with the first three being significantly more common compared to the historical cohort ( $p=$ $0.001, p=0.001$ and $p=0.03$, respectively) (Table 3 ).

With regard to the treatment approach, surgery rate was decreased in the recent cohort $(23.4 \%$ vs. $6.7 \%, p=$ 0.001).

\section{Discussion}

This study describes the changes in the patient characteristics and etiology of bronchiectasis in chidren from a single tertiary reference center beween 2002 and 2019. There were significant differences between the pulmonary function tests at diagnosis and follow-up, underlying etiology, and rate of positive sputum microbiology between the recent and historical cohorts.

Diagnosis of bronchiectasis is often delayed worldwide $[2,3]$. In a study from Italy, Santamaria et al. reported that the median age at diagnosis was 7 years though the children were symptomatic from the median age of 6 months [10]. In developed countries late referral to specialist or misdiagnoses are the most common reasons for diagnostic delay. In the our recent cohort median age at diagnosis was 7 years which was same with our historical cohort. Three different studies from Turkey and studies from indigenous populations also reported similar age at diagnosis (6. 2-8.5 years) [27-30]. In developing countries lack of resources and difficulty in accessing medical services are the most probable causes for the delay in diagnosis [31].

Spirometry results of bronchiectatic patients differ between the developed and developing countries. Lung functions of children from the developed countries were normal or near normal on diagnosis and stayed stable longitudinally [10, 32, 33]. In a study conducted among 991 PCD patients from International PCD cohort, mean $\mathrm{FEV}_{1}$ and mean FVC were lower than the mean reference values in all age groups with best lung function in children aged 6-9 years and the worst in adults [34]. Patients diagnosed at an early age had better lung function

Table 2 Underlying etiologies for non-CF patients

\begin{tabular}{|c|c|c|c|}
\hline & Historical Cohort 1987-2001 & Recent Cohort 2002-2019 & $p$ value \\
\hline Idiopathic & $42(37.8)$ & $20(19.2)$ & 0.03 \\
\hline Postinfectious & $33(29.7)$ & $27(26)$ & 0.43 \\
\hline Immunodeficiencies & $17(15.3)$ & $18(17.3)$ & 0.69 \\
\hline PCD & $7(6.3)$ & $34(32.7)$ & 0.001 \\
\hline Asthma & $5(4.5)$ & $3(2.9)$ & 0.72 \\
\hline Foreign body aspirations & $4(3.6)$ & 0 & NA \\
\hline Others & $3(2.7)$ & $3(2.9)$ & 0.64 \\
\hline Esophageal atresia-tracheoesophageal fistula & $3(2.7)$ & $1(0.9)$ & \\
\hline Cardiac diseases & 0 & $2(2)$ & \\
\hline
\end{tabular}

Values in parentheses are percentages

NA not applicable 
Table 3 Distribution of microorganisms in sputum culture

\begin{tabular}{llll}
\hline & Historical Cohort 1987-2001 & Recent Cohort 2002-2019 & $p$ value \\
\hline None & $53.1 \%$ & $22.1 \%$ & $<0.001$ \\
Hemophilus influenza & $38.5 \%$ & $71.8 \%$ & $<0.001$ \\
Streptococcus pneumonia & $23.0 \%$ & $47.1 \%$ & $<0.001$ \\
Staphylococcus aureus & $16.9 \%$ & $11.5 \%$ & 0.70 \\
Pseudomonas aeruginosa & $10.8 \%$ & $11.5 \%$ & 0.17 \\
Moraxella catarrhalis & $2.7 \%$ & $14.4 \%$ & 0.002 \\
Acinetobacter spp. & None & $3.8 \%$ & $\mathrm{NA}$ \\
Stenotrophomonas maltophilia & None & $3.8 \%$ & $\mathrm{NA}$ \\
Klebsiella pneumonia & $4.6 \%$ & $1.9 \%$ & 0.44 \\
\hline
\end{tabular}

Values are percentages

NA not applicable

and milder disease [32]. A follow-up study enrolling Alaskan Native children showed that patients with bronchiectasis had significantly lower $\mathrm{FEV}_{1} / \mathrm{FVC}$ ratios than the chronic suppurative pulmonary patients without bronchiectasis [35]. In our recent cohort, baseline $\mathrm{FEV}_{1}$ values were higher compared to the historical cohort. Although, there was an increase in $\mathrm{FEV}_{1}$ during the follow-up in the historical cohort, there was no change in the recent cohort. Early and intensive treatment of bronchiectasis has been shown to prevent decline in $\mathrm{FEV}_{1}[1,18,32]$. Kapur et al. also reported that pulmonary functions remained stable in non-CF bronchiectasis patients with a mean $\mathrm{FEV}_{1}$ of $76.8 \pm 20.1 \%$ of predicted after 5 years follow up [32].

Rate of clubbing was significantly lower in the recent cohort compared to the historical cohort supporting the presence of a milder form of bronchiectasis. Rate of clubbing in non-CF bronchiectasis varies with a ratio of 20. $7-52 \%$ and it is more common in developing countries [29, 36, 37]. In a study conducted in non-CF bronchiectatic patients, $52 \%$ had digital clubbing and patients with digital clubbing had more extensive bronchiectasis; no association with pulmonary function tests were seen [37]. In the recent cohort, there was no association with the clubbing of the fingers and lung functions or severity of the bronchiectasis. Although the age of diagnosis did not differ, presence of better baseline pulmonary functions, decreased incidence of clubbing and decrease in follow-up exacerbation rates compared to baseline rates may suggest patients in the recent cohort had a milder form of bronchiectasis. Possible explanations for these changes may be due to increased annual income, better vaccine coverage, earlier recognition and referral and improved access to health care.

In children with bronchiectasis, an underlying disease process is identified in $63 \%$ of cases as shown in a systematic review of 12 studies including 989 children [5]. Previous pneumonia (17\%), primary immunodeficiency (16\%), recurrent aspiration, including inhaled foreign body (10\%), and PCD (9\%) are among the most common underlying etiologies. In developed contries, immunodeficiency is more commonly observed as the underlying disease in $9-34 \%$ of patient with bronchiectasis $[4,8-13,30$, $37,38]$. In developing countries bronchiectasis consequent to previous infection is more common, is the cause in 17 to $28 \%$ of cases [27-29, 39]. An important difference between developed and developing countries in terms of etiology is PCD which is higher in developed countries (15$23.8 \%)[10,12]$. In our recent cohort, $32.7 \%$ of patients with non-CF bronchiectasis were diagnosed as PCD which was significantly higher compared to the historical cohort. Bahceci et al. reviewed 110 non-CF BE patients between 200 5-2015 and compared them with their previous data for underlying etiology. They reported that the frequency of asthma and tuberculosis decreased but PCD (26.4\%) and primary immune deficiency had increased in 10 years [28]. Underlying etiologies of non-CF bronchiectasis can be detected due to increased availability of diagnostic testing. In our cohort only in $19.2 \%$ of the patients underlying etiology could not be identified which is similar with the studies from developed countries [12, 13]. Recent developments in diagnostic testing such as nasal nitric oxide, electron microscopy, high speed videomicroscopy, immunoflorescence and genetic analysis enabled an accurate diagnosis of PCD earlier.

Bronchiectasis has been reported to have multilobar involvement in most pediatric studies [10, 12, 27, 29, 36]. Kapur et al. reported $73 \%$ children as having bilateral disease in their cohort of 52 children [32]. Multilobar disease predominated with a rate of $71 \%$ in a study from Saudi Arabia [36]. In our study group, multilobar involvement was lower. Although, there was a trend in involvement of lobes (less multilobar, more bilobar) between the historical and the recent cohorts, it did not reach statistical significance.

The British Thoracic Society bronchiectasis guideline emphasizes microbiological assessment for evaluating airway colonisation and infection [40]. Although there is 
limited data from developing countries, distribution of micro-organisms seems to be similar worldwide $[1,17,31$, 36, 39]. Studies from developed countries showed that $H$. influenzae, S. pneumoniae and M. catarrhalis are the major pathogens, whereas patients were colonized with Pseudomonas aeruginosa in 5-16\% of children [41]. In our recent cohort, $22.1 \%$ patients had negative sputum cultures, which was significantly lower compared to the historical cohort. Identification of H. influenzae, S. pneumoniae and M. catarrhalis in sputum cultures were increased in the recent cohort. Number of positive sputum cultures may be due to the better qualified staff and equipment in microbiology laboratories leading to more accurate laboratory identification of the microbiology results.

This study had two limitations; it was conducted in a single reference center and as a tertiary referral centre, many patients with suspicion of PCD were referred to our center which might have caused the higher proportion of PCD patients.

\section{Conclusion}

This study highlights the changing underlying aetiology and/or better diagnostic testing of pediatric non-CF bronchiectasis in a developing country setting. We have demonstrated a better lung function results, higher incidence of PCD, decreased incidence of idiopathic cases. An early diagnosis of underlying etiology is essential not only to improve the course and prognosis of disease, but also to prevent a progressive decline in lung function. As the availability of non-invasive and effective diagnostic technologies increase, the detection rate of underlying etiology will increase and improve the outcomes of nonCF bronchiectasis.

\section{Abbreviations}

CF: Cystic fibrosis; PCD: Primary ciliary dyskinesia; HRCT: High resolution chest tomography; TST: Tuberculin skin test; FVC: Forced vital capasity;

FEV1: Forced expiratory volume in $1 \mathrm{~s}$

\section{Acknowledgements}

Not applicable.

\section{Authors' contributions}

$E E E, Y G, R E, F K$, BK researched literature and conceived the study. EEE, EA, $\mathrm{NBI}, \mathrm{PE}, \mathrm{CYY}, \mathrm{AK}$ were involved in protocol development, gaining ethical approval, patient recruitment and data analysis was performed by EEE and BK. EEE wrote the first draft of the manuscript. All authors reviewed and edited the manuscript and approved the final version of the manuscript.

\section{Funding}

The authors did not receive any specific grant from funding agencies in the public or commercial sectors.

\section{Availability of data and materials}

The datasets used and/or analysed during the current study are available from the corresponding author on reasonable request.

\section{Ethics approval and consent to participate}

The study was approved by the Ethical Committee of Marmara University Medical Faculty and written informed consent was taken from the patients families.
Consent for publication

Not applicable.

\section{Competing interests}

The authors declare that they have no competing interests.

\section{Author details}

${ }^{1}$ Division of Pediatric Pulmonology, Marmara University, School of Medicine, Istanbul, Turkey. ${ }^{2}$ Division of Pediatric Pulmonology, Maltepe University, School of Medicine, Istanbul, Turkey.

Received: 20 September 2019 Accepted: 11 June 2020

Published online: 16 June 2020

\section{References}

1. Chang AB, Bush A, Grimwood K. Bronchiectasis in children: diagnosis and treatment. Lancet. 2018;392(10150):866-79.

2. Goyal V, Grimwood K, Marchant J, Masters IB, Chang AB. Pediatric bronchiectasis: no longer an orphan disease. Pediatr Pulmonol. 2016;51:450-69.

3. McCallum GB, Binks MJ. The epidemiology of chronic suppurative lung disease and bronchiectasis in children and adolescents. Front Pediatr. 2017; 5:27.

4. Twiss J, Metcalfe R, Edwards E, Byrnes C. New Zealand national incidence of 2005 bronchiectasis "too high" for a developed country. Arch Dis Child. 2005;90:737-40.

5. Brower KS, Del Vecchio MT, Aronoff SC. The etiologies of non-CF bronchiectasis in childhood: a systematic review of 989 subjects. BMC Pediatr. 2014;14:4

6. Chang AB, Masel JP, Boyce NC, Wheaton G, Torzillo PJ. Non-CF bronchiectasis: clinical and HRCT evaluation. Pediatr Pulmonol. 2003;35:477-83.

7. Singleton RJ, Morris A, Redding A, Poll J, Holck P, Martinez P, Kruse D, Bulkow LR, Petersen KM, Lewis C. Bronchiectasis in Alaska native children: cases and clinical courses. Pediatr Pulmonol. 2000;29:182-7.

8. Zaid AA, Elnazir B, Greally P. A decade of non-cystic fibrosis bronchiectasis 1996-2006. Ir Med J. 2010;103:77-9.

9. Kapur N, Grimwood K, Masters IB, Morris PS, Chang AB. Lower airway microbiology and cellularity in children with newly diagnosed non-CF bronchiectasis. Pediatr Pulmonol. 2012:47:300-7.

10. Santamaria F, Montella S, Pifferi M, Ragazzo V, De Stefano S, De Paulis N, Maglione M, Boner AL. A descriptive study of non-cystic fibrosis bronchiectasis in a pediatric population from central and southern Italy. Respiration. 2009:77:160-5.

11. Nikolaizik WH, Warner JO. Aetiology of chronic suppurative lung disease. Arch Dis Child. 1994:70:141-2.

12. Li AM, Sonnappa S, Lex C, Wong E, Zacharasiewicz A, Bush A, Jaffe A. NonCF bronchiectasis: does knowing the aetiology lead to changes in management? Eur Respir J. 2005;26:8-14.

13. Eastham KM, Fall AJ, Mitchell L, Spencer DA. The need to redefine noncystic fibrosis bronchiectasis in childhood. Thorax. 2004;59:324-7.

14. Kumar A, Lodha R, Kumar P, Kabra SK. Non-cystic fibrosis bronchiectasis in children: clinical profile, etiology and outcome. Indian Pediatr. 2015;52:35-7.

15. Quint JK, Millett ER, Joshi M, Navaratnam V, Thomas SL, Hurst JR, Smeeth L, Brown JS. Changes in the incidence, prevalence and mortality of bronchiectasis in the UK from 2004 to 2013: a population-based cohort study. Eur Respir J. 2016:47:186-93.

16. Diel R, Ewig S, Blaas S, Jacob C, Juelich F, Korfmann G, Sohrab S, Sutharsan $S$, Rademacher J. Incidence of patients with non-cystic fibrosis bronchiectasis in Germany-a healthcare insurance claims data analysis. Respir Med. 2019;151:121-7.

17. Karadag B, Karakoc F, Ersu R, Kut A, Bakac S, Dagli E. Non-cystic-fibrosis bronchiectasis in children: a persisting problem in developing countries. Respiration. 2005;72:233-8.

18. Chang AB, Bell SC, Torzillo PJ, King PT, Maguire GP, Byrnes CA, Holland AE, O'Mara P, Grimwood K, extended voting group. Chronic suppurative lung disease and bronchiectasis in children and adults in Australia and new Zealand Thoracic Society of Australia and new Zealand guidelines. Med J Aust. 2015;202:21-3.

19. McGuinness G, Naidich DP, Leitman BS, McCauley DI. Bronchiectasis: CT evaluation. Am J Roentgenol. 1993;160:253-9. 
20. Kapur N, Masters IB, Morris PS, Galligan J, Ware R, Chang AB. Defining pulmonary exacerbation in children with non-cystic fibrosis bronchiectasis. Pediatr Pulmonol. 2012;47:68-75.

21. Valery PC, Morris PS, Byrnes CA, et al. Long-term azithromycin for indigenous children with non-cystic-fibrosis bronchiectasis or chronic suppurative lung disease (bronchiectasis intervention study): a multicentre, double-blind, randomised controlled trial. Lancet Respir Med. 2013;1:610-20.

22. Leigh MW, Hazucha MJ, Chawla KK, Baker BR, Shapiro AJ, Brown DE, Lavange LM, Horton BJ, Qaqish B, Carson JL, et al. Standardizing nasal nitric oxide measurement as a test for primary ciliary dyskinesia. Ann Am Thorac Soc. 2013;10:574-81.

23. Global Initiative for Asthma. GINA report, global strategy for asthma management and prevention. Revised 2019. Available at: www.ginasthma.org.

24. Poglar G, Promadhat V. Pulmonary function testing in children: techniques and standards. Philadelphia: Saunders; 1971.

25. Pellegrino R, Viegi G, Brusasco V, Crapo RO, Burgos F, Casaburi R, Coates A, Van der Grinten CP, Gustafsson P, Hankinson J, et al. Interpretative strategies for lung function tests. Eur Respir J. 2005;26:948-68.

26. Atag E, Hjeij R, Ikizoğlu NB, Ergenekon P, Yegit CY, Gokdemir Y, et al. Immunofluorescense staining results in respiratory epithelial cells of patients with suspected PCD from Marmara University. ERJ. 2019;54(Suppl 63):PA386.

27. Karakoc GB, Yilmaz M, Altintas DU, Kendirli SG. Bronchiectasis: still a problem. Pediatr Pulmonol. 2001;32:175-8.

28. Bahçeci S, Karaman S, Nacaroğlu HT, Yazıcı S, Girit S, Ünsal-Karkıner S, Can D. Changing epidemiology of non-cystic fibrosis bronchiectasis. Turk J Pediatr. 2016;58:19-26.

29. Satırer O, Mete Yesil A, Emiralioglu N, Tugcu GD, Yalıı E, Dogru D, Kiper N, Ozcelik U. A review of the etiology and clinical presentation of non-cystic fibrosis bronchiectasis: a tertiary care experience. Respir Med. 2018;137:35-9.

30. Munro KA, Reed PW, Joyce H, Perry D, Twiss J, Byrnes CA, Edwards EA. Do New Zealand children with non-cystic fibrosis bronchiectasis show disease progression? Pediatr Pulmonol. 2011;46:131-8.

31. Kapur N, Karadag B. Differences and similarities in non-cystic fibrosis bronchiectasis between developing and affluent countries. Paediatr Respir Rev. 2011:12:91-6.

32. Kapur N, Masters IB, Chang AB. Longitudinal growth and lung function in pediatric non-CF bronchiectasis-what influences lung function stability? Chest. 2010;138:158-64.

33. Bastardo CM, Sonnappa S, Stanojevic S, Navarro A, Lopez PM, Jaffe A, Bush A. Non-cystic fibrosis bronchiectasis in childhood: longitudinal growth and lung function. Thorax. 2009;64:246-51.

34. Halbeisen FS, Goutaki M, Spycher BD, Amirav I, Behan L, Boon M, et al. Lung function in patients with Primary Ciliary Dyskinesia: an PCD Cohort study. Eur Respir J. 2018;52(2):1801040.

35. Kinghorn B, Singleton R, McCallum GB, Bulkow L, Grimwood K, Hermann L, Chang $A B$, Redding $G$. Clinical course of chronic suppurative lung disease and bronchiectasis in Alaska native children. Pediatr Pulmonol. 2018;53: 1662-9.

36. Banjar HH. Clinical profile of Saudi children with bronchiectasis. Indian J Pediatr. 2007:74:149-52.

37. Edwards EA, Metcalfe R, Milne DG, Thompson J, Byrnes CA. Retrospective review of children presenting with non cystic fibrosis bronchiectasis: HRCT features and clinical relationships. Pediatr Pulmonol. 2003;36:87-93.

38. Kapur N, Masters IB, Chang AB. Exacerbations in noncystic fibrosis bronchiectasis: clinical features and investigations. Respir Med. 2009;103: $1681-7$.

39. Lai SH, Wong KS, Liao SL. Clinical analysis of bronchiectasis in Taiwanese children. Chang Gung Med J. 2004;27:122-8.

40. Pasteur MC, Bilton D, Hill AT. British Thoracic Society quideline for non-CF bronchiectasis. Thorax. 2010;65(Suppl. 1):i1-58.

41. Pizzutto SJ, Hare KM, Upham JW. Bronchiectasis in children: current concepts in immunology and microbiology. Front Pediatr. 2017;5:123.

\section{Publisher's Note}

Springer Nature remains neutral with regard to jurisdictional claims in published maps and institutional affiliations.

\section{Ready to submit your research? Choose BMC and benefit from}

- fast, convenient online submission

- thorough peer review by experienced researchers in your field

- rapid publication on acceptance

- support for research data, including large and complex data types

- gold Open Access which fosters wider collaboration and increased citations

- maximum visibility for your research: over $100 \mathrm{M}$ website views per year

At BMC, research is always in progress.

Learn more biomedcentral.com/submissions 